\title{
RISK ASSESSMENT OF IMPLEMENTING INNOVATIVE PROJECTS IN ENTERPRISES USING ARTIFICIAL NEURAL NETWORKS
}

\author{
Roman M. Kachalov \\ Central Economic Mathematical Institute of the Russian Academy of Sciences, Moscow, Russian Federation; \\ Dubna State University, Dubna, Russian Federation \\ Yulia A. Sleptsova \\ Central Economic Mathematical Institute of the Russian Academy of Sciences, Moscow, Russian Federation; \\ Dubna State University, Dubna, Russian Federation
}

\author{
Yan V. Shokin \\ Dubna State University, Dubna, Russian Federation
}

\begin{abstract}
The purpose of this paper is to shed new light on the issue of risk assessment in implementing innovative projects. Based on the ideas of George Kleiner and artificial neural network tools, this paper interprets the possibility of completing successful innovation projects in terms of risk management. The argument is buttressed with a case study of a set of Russian enterprises implementing innovative projects. Successful completion of an innovation project depends on a number of risk factors identified at the project start. The identified set of risk factors should include both innovative risk factors and non-innovative risk factors. This fact was established during the cluster analysis of the available data. The paper excludes from consideration the anti-risk management impact and does not take into account the weights of various risk factors in the problem formalization. The practical application of the results of the study is decision makers' quest to strike a visual interpretation of the final data with a small number of possible scenarios that differ significantly from each other. This research contributes significantly to the literature on the risk assessment model of innovative projects. The failure of managers to balance the assessment of innovative risk factors and non- innovative risk factors is exposed as the root cause of the unsuccessful completion of innovative projects.
\end{abstract}

Key words: risk factors, risk assessment, innovative project, artificial neural network, cluster analysis.

Citation. Kachalov R.M., Sleptsova Yu.A., Shokin Ya.V. Risk Assessment of Implementing Innovative Projects in Enterprises Using Artificial Neural Networks. Journal of Volgograd State University. Economics, 2019, vol. 21,

\section{ОЦЕНКА РИСКА РЕАЛИЗАЦИИ ИННОВАЦИОННЫХ ПРОЕКТОВ ПРЕДПРИЯТИЙ С ПОМОЩЬЮ ИСКУССТВЕННЫХ НЕЙРОННЫХ СЕТЕЙ}

\author{
Роман Михайлович Качалов
}

Центральный экономико-математический институт РАН, г. Москва, Российская Федерация; Государственный университет «Дубна», г. Дубна, Российская Федерация

\section{Юлия Анатольевна Слепцова}

Центральный экономико-математический институт РАН, г. Москва, Российская Федерация; Государственный университет «Дубна», г. Дубна, Российская Федерация 


\section{УПРАВЛЕНИЕ ЭКОНОМИЧЕСКИМ РАЗВИТИЕМ}

\section{Ян Вячеславович Шокин}

Государственный университет «Дубна», г. Дубна, Российская Федерация

Аннотация. Цель данной статьи - предложить новый подход к оценке риска при реализации инновационных проектов. Используя системную экономическую теорию и инструменты искусственных нейронных сетей, мы рассмотрели возможность успешного завершения инновационных проектов с точки зрения управления уровнем риска. Исследовано некоторое множество российских предприятий, реализующих инновационные проекты. В ходе исследования была подтверждена гипотеза о том, что успешное завершение инновационного проекта зависит от системы управления уровнем риска, в частности, от идентификации факторов риска в начале выполнения проекта. Этот факт был установлен в ходе кластерного анализа имеющихся данных. Ограничения / последствия исследования: не учитывается вес различных факторов риска при формализации проблемы. Практическое применение результатов исследования заключается в возможности визуальной интерпретации лицами, принимающими решения, окончательных данных с небольшим числом возможных сценариев, которые значительно отличаются друг от друга. Оригинальность исследования заключается в сочетании применения системной экономической теории и аппарата обучающихся искусственных нейронных сетей к оценке риска невыполнения инновационных проектов.

Ключевые слова: факторы риска, оценка риска, инновационный проект, искусственная нейронная сеть, кластерный анализ.

Цитирование. Качалов Р. М., Слепцова Ю. А., Шокин Я. В. Оценка риска реализации инновационных проектов предприятий с помощью искусственных нейронных сетей // Вестник Волгоградского государственного университета. Экономика. - 2019. - Т. 21, № 4. -C. 171-181. -DOI: https://doi.org/10.15688/ek.jvolsu.2019.4.17

\section{Введение}

Недостаток прикладных методических рекомендаций, опирающихся на достижения экономической науки, затрудняет применение релевантных методов управления уровнем риска инновационных проектов руководителями предприятий. Для полноценного развития научного экономического знания недостаточно ограничиваться описанием выявляемых факторов экономического риска, присущих таким проектам. Большую ценность могут представлять исследования в области инструментальных возможностей прогнозирования успешности реализации проектов, инициируемых на предприятиях. При этом важно, чтобы эти исследования базировались на теоретическом аппарате поведенческой экономики и ряде прикладных методов теории прогнозирования. Для решения подобных задач предложена концептуальная модель обоснования и принятия экономических решений с учетом факторов риска и последующим прогнозированием их возможных позитивных и негативных последствий на основе применения инструментария искусственной нейронной сети.

Цель настоящего исследования, выполняемого при финансовой поддержке Российского фонда фундаментальных исследований (проект № 18-010-01042), - разработка обу- чающейся нейросетевой модели оценки влияния факторов риска на управленческие решения в инновационной сфере.

В данной работе предложено решение классификационной задачи для множества факторов экономического риска и событийпоследствий их реализации. При этом в рамках исследования предполагается, что управление уровнем риска реализуемого проекта включает в себя общие принципы, на основе которых в дальнейшем строятся технологические и бизнес-процессы, осуществляются коммуникации не только внутри предприятия, но и во внешней среде. Таким образом, можно считать, что к основным этапам анализа риска нереализации инновационных проектов предприятия (внутренний контроль и мониторинг факторов риска) добавляется еще и разработка антирисковых управленческих воздействий по снижению уровня риска недостижения целей инициированных проектов [Качалов, 2012; Качалов и др., 2014].

При учете сложности формализации задачи управления уровнем риска на конкретном предприятии и отсутствие больших массивов данных об ошибках, допущенных при внедрении инноваций, а также принятии во внимание уникальности бизнес-процессов на каждом предприятии, для анализа был выбран инструментарий искусственных нейронных се- 
тей. Преимущества выбранного математического аппарата состоят в том, что процесс исследования с использованием нейронных сетей значительно упрощен, поскольку нейронная сеть не требует отдельного алгоритма для решения каждой конкретной задачи и способна к обучению в процессе моделирования.

В данном исследовании в качестве алгоритма искусственной нейронной сети был использован метод обучения без учителя, то есть результат обучения зависит только от структуры входных данных. В процессе обучения сети устанавливается связь между набором начальных и конечных данных. Отсутствие стандартных подходов и методик по использованию в тех или иных случаях нейронных сетей определенного вида, а также требований к структуре таких сетей может сдерживать эффективное применение моделирования с их помощью. При этом следует отметить, что на данном этапе исследования оценка стратегических альтернатив не производилась.

\section{Система управления уровнем риска предприятия}

Согласно системной экономической теории [Клейнер, 2013] выделяется четыре типа систем: объектные, проектные, процессные и средовые. Рассматривая предприятие как систему и учитывая последние рекомендации организаций, объединяющих специалистов по управлению риском, например [COSO..., 2017], можно предположить, что предприятие, с точки зрения системы управления уровнем риска можно представить в виде взаимодействия четырех подсистем тех же видов: объектного, проектного, процессного и средового [Качалов и др., 2015].

Объектная подсистема предприятия включает в себя как материальные, так и нематериальные активы. При этом факторы риска объектной подсистемы могут быть выявлены как на этапе добавления новых активов в ходе реализации проекта, например, в процессе их покупки, строительства или разработки, так и в процессе их использования, или эксплуатации.

В проектную подсистему предприятия входят все инициированные и реализуемые проекты. Основные группы факторов риска, выявляемые в этой подсистеме, обусловлены тем, что каждый проект обладает своей целью, утвержденными сроками и бюджетом. В связи с этим при инициировании инновационных проектов в рамках этой подсистемы может проводиться оценка стратегических альтернатив, анализ условий деятельности предприятия и выявление факторов риска недостижения цели проекта, нарушения запланированных сроков реализации проекта или превышения утвержденного бюджета.

Проиессная подсистема предприятия включает в себя все технологические и бизнес-процессы, в которые вовлечены сотрудники, отдельных подразделений или всего предприятия в целом и которые характеризуются устойчивостью и многократным повторением. Совокупность процессов, составляющих процессную подсистему, направлена на преобразование материальных, трудовых и информационных ресурсов в экономические результаты предприятия - продукцию и услуги. Факторы риска этой подсистемы связаны с авариями, сбоями или иными нарушениями ритмичности процессов.

Средовая подсистема предприятия определяется характеристиками принятого типа корпоративного управления и сложившейся культурой предприятия в части приверженности основным ценностям, иными словами, эта подсистема представляет собой сложившиеся культурные традиции, социальные условия и другие неформальные институты в пределах предприятия, а также действующее законодательство всех уровней, регулирующее деятельность по производству и реализации профильной продукции предприятия. Факторы риска этой подсистемы могут быть выявлены как во внешней, так и во внутренней среде предприятий. Внутренние проблемы могут порождаться оппортунистическим поведением отдельных сотрудников и даже целых групп внутри трудового коллектива, их сопротивлением новшествам, а также плохо подготовленными управленческими решениями со стороны руководства. Внешние факторы риска обусловливаются технологическими, институциональными и культурными ограничениями страны [Полтерович, 2016]. 


\section{Факторы риска}

инновационного развития предприятия

В ряде зарубежных исследований, например в [Kapoor et al., 2013], подчеркивалось, что управление инновационными проектами на предприятии имеет сложную структуру, может вызвать цепную реакцию на изменения во всех бизнес-процессах предприятия и вовлечь в инновационные процессы множество сотрудников и участников, и при определенных условиях такой проект может быть спланирован заранее довольно точно. В работе [Gawer et al., 2014] исследована роль действующих лиц в создании инновационных проектов. Было показано, что предприятия, разрабатывающие инновационные продукты и новый дизайн товаропроводящих сетей, стремятся занять лидирующие позиции в системе и организовать ее. В этом случае фактор риска неудачных коммуникаций является неотъемлемой частью любого стратегического сотрудничества между предприятиями и организациями. Например, в работе [Das et al., 2001] была подчеркнута высокая вероятность негативных последствий неудачного сотрудничества.

Исследования инновационных предприятий, входящих в национальные инновационные системы, выявили и смогли дать некоторое представление о стратегических и коммуникационных факторах риска [Das et al., 1996]. Однако эта точка зрения имеет некоторые ограничения при рассмотрении основных характеристик современных инновационных систем. Системы состоят из разнонаправленных отношений между организациями, а также руководителей и специалистов различных предприятий с высокой степенью многосторонней зависимости друг от друга. Эти обстоятельства отличают ситуацию от традиционных цепочек, основанных на двусторонних отношениях [Walley, 2007], поскольку современные предприятия включены в сети многосторонних отношений [Boland et al., 2007; Schilling et al., 2007] для совместного создания инновационных продуктов и услуг.

В современной экономике успех инновационного предприятия зависит не только от его собственных усилий, но и от степени сотрудничества между предприятиями. Успех может быть достигнут благодаря многостороннему сотрудничеству в рамках специально организованного инновационного комплекса предприятий [Iansiti et al., 2002; Gulati et al., 2000].

Факторы риска инновационного проекта имеют некоторые особенности. Так, фактор риска можно отнести к инновационным, если он напрямую связан с созданием, дальнейшим производством и распространением инновационного продукта или услуги. Другой особенностью инновационного фактора риска можно считать отсутствие методов управления уровнем этого вида риска как в деятельности отдельного предприятия, так и в доступных источниках информации [Качалов и др., 2014].

Недостаток информации о негативных последствиях тех или иных управленческих решений при внедрении инновационных проектов затрудняет формализацию и решение задачи построения математических моделей оценки уровня соответствующего вида риска. В связи с этим для моделирования был выбран аппарат искусственных нейронных сетей.

\section{Моделирование оценки уровня риска реализации инновационного проекта \\ с помощью искусственной нейронной сети}

Использование искусственных нейронных сетей расширяет совокупность существующих формальных моделей принятия решений [Estimating Efficient..., 2012; Learning and Clustering..., 2014] и управления уровнем риска. Основная роль применения искусственных нейронных сетей для управления уровнем риска состоит в формулировании нового подхода к разработке управленческих решений в условиях реализации инновационных проектов [Романовский и др., 2010]. Искусственная нейронная сеть при моделировании процессов управления уровнем риска, как показала практика, позволяет корректно описывать сложные ситуации возникновения экономического риска и обеспечивает независимый механизм прогноза успешности анализируемых проектов [Misra et al., 2010]. Таким образом оказывается возможным сравнивать исторические данные о факторах риска, выявленных в аналогичных проектах, с факторами риска новых проектов [Sarcia et al., 2007]. 
Оценка риска высокотехнологичных проектов представляет собой сложный процесс, включающий оценку характеристик различных факторов риска и выявление нелинейных зависимостей между существенными факторами риска и результатами измерения их значимости [Zhang et al., 1998; Sarcia et al., 2006]. Для оценки уровня риска в таких проектах применялись как качественные, так и количественные методы. С помощью искусственных нейронных сетей можно с высокой точностью классифицировать существенные факторы риска и прогнозировать нелинейные зависимости [Pukala, 2016; Jiang, 2009], а также риск неудачного завершения проекта [Analysis of the Effect..., 2014].

В предпринятом нами исследовании проводится оценка риска недостижения основной стратегической цели на примере ряда предприятий, реализующих инновационные проекты. Для этого была построена искусственная нейронная сеть, использующая данные о ряде российских предприятий. О выборке этих предприятий и их проектов (использовались данные о 75 проектах) известно, что от начала реализации проектов прошло около 10 лет. Некоторые завершились неудачей, поэтому для простоты будем считать, что проект завершен неудачно, если он не может быть продолжен по любой причине. Другие же были успешно реализованы. В этом случае успешно реализованный проект означает, что какаято доля в проекте была продана профильному инвестору, что позволило не только компенсировать все затраты по проекту, но и получить некоторый доход от этой продажи. Поскольку по некоторым из проектов использованной выборки нет однозначной информации о том, можно ли их считать реализованными успешно, либо неуспешно, было принято решение в дальнейшем анализе использовать данные лишь о тех проектах, по которым имеется информация о результатах выполнения.

На начальной стадии выполнения проекта и в последующие периоды аудиторы и руководители предприятия идентифицируют различные факторы риска, препятствующие достижению цели проекта. Если предприятие прилагает определенные усилия, то антирисковые мероприятия сводят к минимуму риски неудачи. Информация о выявленных фак- торах риска и их характеристиках - как для успешных, так и для неудачных проектов была загружена в качестве входных параметров в нейронную сеть. При этом было выделено 33 разновидности факторов риска (например, операционные, маркетинговые, финансовые, технологические риски и т. п.). Кроме того, совокупность выявленных факторов риска подразделялась на 4 группы (согласно уже упоминавшейся системной экономической теории Г.Б. Клейнера) на факторы риска, относящиеся, соответственно, к объектной, процессной, проектной или средовой подсистеме.

Для формализации описания оценки риска инновационного проекта моделируется искусственная нейронная сеть, на первом этапе моделирования вводятся обозначения переменных (X1, X2, X3, X4), которые характеризуют некоторое событие как проявление фактора риска соответствующей группы, оказывая существенное влияние на риск недостижения цели проекта (Y):

1. X1 - факторы риска объектной подсистемы предприятия, например, факторы риска неправильного использования ресурсов.

2. X2 - факторы риска процессной подсистемы предприятия, к таким факторам риска могут быть отнесены, в том числе, факторы риска нарушения технологических и бизнес-процессов при реализации проекта.

3. X3 - факторы риска проектной подсистемы предприятия, в эту группу включаются факторы риска технической невозможности достижения целевых параметров инновационной продукции, например, невозможность масштабирования технологии.

4. X4 - факторы риска средовой подсистемы предприятия, например, факторы рыночных рисков, когда рынок не готов к новому продукту - отсутствует техническая инфраструктура или не хватает квалифицированных кадров для производства.

Процесс моделирования искусственной нейронной сети состоял из следующих этапов:

- определение общего числа показателей (в нашем случае факторов риска), способных характеризовать анализируемый проект;

- отсев из общего числа показателей, выбранных на первом этапе, тех, которые можно признать несущественно влияющими на успешное завершение проекта; 


\section{УПРАВЛЕНИЕ ЭКОНОМИЧЕСКИМ РАЗВИТИЕМ}

- формирование обучающей выборки для нейронной сети, включающей значения отобранных на предыдущем этапе показателей за некоторый период времени;

- проверка искусственной нейронной сети на тестовой выборке.

В данном исследовании для моделирования было использовано программное обеспе-åí èå DataBase Deductor Studio Academic 5.3, с помощью которого построена искусственная нейронная сеть, которая имеет два скрытых слоя. Сеть характеризовалась следующими параметрами:

- в качестве функции активации взята стандартная сигмоида с коэффициентом крутизны, равным единице;

- выбран алгоритм распространения ошибки - Back propagation (алгоритм обратного распространения ошибки);

- выборка разбита на обучающее и тестовое подмножества; разбиение произведено в пропорции: 95 (девяносто пять) процентов предприятий включено в обучающее подмножество, а в тестовую выборку вошло 5 (пять) процентов предприятий;
- принятые условия остановки обучения: по числу итераций (эпох) - нет ограничений; средняя нормированная ошибка по обучающему множеству - не выше 0,05 ; средняя нормированная ошибка по тестовому множеству - не выше 0,05; пример считается распознанным, если нормированная ошибка не более 0,05 .

В итоге сеть после примерно 750 итераций (эпох) смогла распознать 77 \% примеров обучающего множества и $100 \%$ тестового, при этом предсказанный сетью выход совпал с выходным значением по выборке в 95,7 \% случаев (неправильно предсказан лишь один пример). Граф полученной нейронной сети представлен на рисунке.

Также был произведен кластерный анализ имеющихся данных с целью выявления устойчивых кластеров проектов по некоторому характерному набору влияющих факторов риска. Для этого в программном продукте DataBase Deductor Studio Academic 5.3. был использован алгоритм ЕМ-кластеризации, в результате которого наилучшим разбиением исходного множества проектов по факторам риска следует признать выделение 3 (трех)

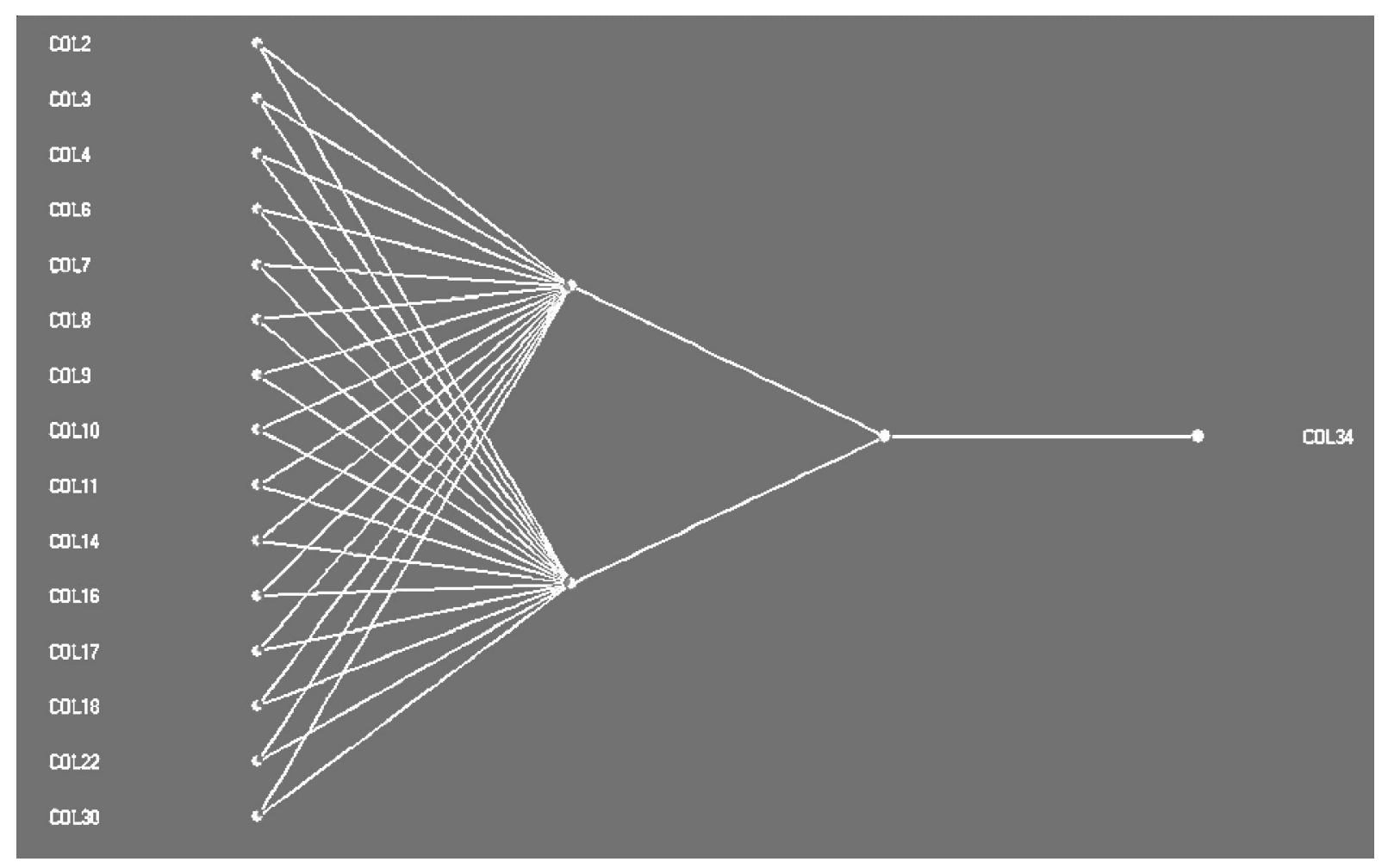

Рисунок. Граф полученной нейросети с двумя скрытыми слоями

Примечание. Составлено авторами при проведении моделирования с использованием пакета DataBase Deductor Studio Academic 5.3. 
кластеров: 0,1 и 2 с наибольшей вероятностью отнесения каждого объекта к тому или иному кластеру. Результаты кластеризации приведены в таблице.

Как видно из таблицы, в кластер 2 в подавляющем большинстве случаев попали проекты, характеризующиеся наименьшим суммарным количеством выделенных факторов риска; при этом жесткой связи с результатом проекта (0 или 1) обнаружено не было. В кластеры же 0 и 1 попали те проекты, в которых суммарное количество выделенных факторов риска оказалось значительно большим, при этом для кластера 0 характерно смещение данных факторов в сторону групп X3 и X4 (факторы риска проектной и средовой подсистем), в то время как для кластера 1 характерно более равномерное распределение факторов риска.

На этом основании можно сделать вывод, что при разработке и реализации инновационных проектов компаниям необходимо особое внимание уделять контролю над факторами риска проектной и средовой подсистем.

\section{Заключение}

В данном исследовании предложен новый подход к системе управления уровнем риска и новая модель оценки уровня риска нереализации инновационного проекта. Построение модели основано на значительном упрощении изучаемой ситуации, при выборе и управлении параметрами модели проводится качественный и количественный анализ оценки уровня риска на сравнительно небольшом количестве проектов за период их реализации. Это было проделано на основе информации о выявленных факторах риска как при инициировании проектов, так и на следующих этапах реализации проектов. Базируясь на системной экономической теории Г.Б. Клейнера, мы разбили все выявленные факторы риска на четыре группы: объектной, процессной, проектной и средовой подсистем. На следующем этапе сформировался набор входных переменных, который был загружен в программную среду для построения нейронной сети, а за-

Таблица

Результаты кластеризации данных, полученных при обучении нейронной сети методом ЕМ-кластеризации

\begin{tabular}{|c|c|c|c|c|c|}
\hline \multicolumn{4}{|c|}{ Факторы риска по типам подсистем } & \multirow{2}{*}{$\begin{array}{c}\text { Бинарный } \\
\text { выход } \\
\text { модели (Y) }\end{array}$} & \multirow[b]{2}{*}{$\begin{array}{c}\text { Номер } \\
\text { кластера }\end{array}$} \\
\hline $\begin{array}{c}\text { Объектная } \\
\text { (X1) }\end{array}$ & $\begin{array}{c}\text { Процессная } \\
\text { (X2) }\end{array}$ & $\begin{array}{c}\text { Проектная } \\
\text { (X3) }\end{array}$ & $\begin{array}{c}\text { Средовая } \\
\text { (X4) }\end{array}$ & & \\
\hline 3 & 1 & 2 & 1 & 1 & $\mathbf{0}$ \\
\hline 1 & 1 & 0 & 0 & 1 & 2 \\
\hline 0 & 3 & 3 & 1 & 1 & 2 \\
\hline 0 & 1 & 0 & 2 & 0 & 2 \\
\hline 0 & 0 & 1 & 2 & 0 & 2 \\
\hline 0 & 0 & 2 & 1 & 0 & 2 \\
\hline 0 & 0 & 1 & 6 & 1 & $\mathbf{0}$ \\
\hline 0 & 0 & 3 & 2 & 1 & 1 \\
\hline 1 & 0 & 3 & 3 & 0 & 1 \\
\hline 3 & 0 & 10 & 3 & 1 & $\mathbf{0}$ \\
\hline 0 & 2 & 9 & 4 & 1 & $\mathbf{0}$ \\
\hline 0 & 1 & 0 & 3 & 1 & 2 \\
\hline 11 & 3 & 5 & 4 & 0 & $\mathbf{0}$ \\
\hline 0 & 0 & 1 & 1 & 0 & 2 \\
\hline 0 & 0 & 2 & 1 & 1 & 2 \\
\hline 1 & 0 & 0 & 1 & 1 & 2 \\
\hline 0 & 2 & 4 & 2 & 1 & 2 \\
\hline 0 & 2 & 2 & 7 & 1 & 0 \\
\hline 0 & 0 & 2 & 2 & 1 & 2 \\
\hline 1 & 5 & 3 & 4 & 0 & 2 \\
\hline 0 & 0 & 2 & 1 & 1 & 1 \\
\hline 0 & 2 & 2 & 3 & 1 & 2 \\
\hline 1 & 4 & 4 & 8 & 1 & 0 \\
\hline
\end{tabular}

Примечание. Составлено авторами. 
тем было оценено правдоподобие генерации выходов модели на тестовой выборке.

Таким образом, нейросетевой подход к моделированию управления уровнем риска позволяет корректно описывать сложные ситуации проявления экономического риска и их характеристики. Кроме того, были применены методы кластеризации для определения нескольких типичных сценариев разработки и завершения инновационного проекта.

Наибольший практический интерес для лиц, принимающих решения в области управления уровнем риска, представляет наглядная интерпретация итоговых данных при небольшом числе возможных сценариев, существенно друг от друга отличающихся.

\section{СПИСОК ЛИТЕРАТУРЫ}

Качалов, Р. М. Динамика риска в процессах инновационного развития предприятий / Р. М. Качалов, Ю. А. Слепцова // Вестник Волгоградского государственного университета. Серия 3, Экономика. Экология. - 2014. № 4 (27). - C. 72-86. - DOI: http://dx.doi.org/ 10.15688/jvolsu3.2014.4.8.

Качалов, Р. М. Структурирование системно-экономического пространства предприятия в задачах управления уровнем риска / Р. М. Качалов, Ю. А. Слепцова // Российский журнал менеджмента. -2015. - Т. 13, № 4. - С. 69-84.

Качалов, Р. М. Управление экономическим риском: теоретические основы и приложения / Р. М. Качалов. - СПб. : Нестор-История, 2012. - 288 с.

Клейнер, Г. Б. Системная экономика как платформа развития современной экономической теории / Г. Б. Клейнер // Вопросы экономики. 2013. - № 6. - C. 4-28. - DOI: https://doi.org/ 10.32609/0042-8736-2013-6-4-28.

Полтерович, В. М. Институты догоняющего развития (к проекту новой модели экономического развития России) / В. М. Полтерович // Экономические и социальные перемены: факты, тенденции, прогноз. - 2016. - № 5 (47). - С. 34-56.

Романовский, А. В. Нейроэкономика и ее интеграция в экономическую науку / А. В. Романовский, Я. В. Шокин // Экономические науки. 2010. - № 4. - C. 42-45.

Analysis of the Effect of Risk Management Practices on the Performance of New Product Development Programs / J. Oehmen [et al.] // Technovation, Elsevier Ltd. - 2014. - Vol. 34, № 8. - P. 441-453. - DOI: https://doi.org/10.1016/ j.technovation. 2013.12.005.
Boland, J. R. J. Wakes of Innovation in Project Networks: The Case of Digital 3-D Representations in Architecture, Engineering, and Construction / J. R. J. Boland, K. Lyytinen, Y. Yoo // Organization Science. - 2007. № 18 (4). - P. 631-647. - DOI: https://doi.org/ 10.1287/orsc. 1070.0304 .

COSO: Enterprise Risk Management - Integrating with Strategy and Performance (2017). - Electronic text data. - Mode of access: https://www.coso. org/Pages/erm.aspx (date of access: 05.06.2019).

Das, T. Risk Types and Inter-Firm Alliance Structures / T. Das, B. Teng // Journal of Management Studies. - 1996. - № 33 (6). P. 827-843. - DOI: https://doi.org/10.1111/j.1467-6486.1996.tb00174.x.

Das, T. Trust, Control, and Risk in Strategic Alliances: An Integrated Framework / T. Das, B. Teng // Organization Studies. - 2001. - № 22 (2). P. 251-283.

Estimating Efficient Value of Controllable Variable Using an Adaptive Neural Network Algorithm: Case of a Railway System / A. Azadeh [et al.] // Journal of Scientific and Industrial Research. 2012. - № 71 (1). - P. 45-50.

Gawer, A. Industry platforms and ecosystem innovation / A. Gawer, M. Gusumano // Journal of Product Innovation Management. - 2014. № 31 (3). - P. 417-433. - DOI: https://doi.org/ 10.1111/jpim. 12105.

Gulati, R. Strategic Networks / R. Gulati, N. Nohria, A. Zaheer // Strategic Management Journal. 2000. - №21(3). -P.203-215.-DOI: https://doi.org/ 10.1002/(SICI)1097-0266(200003)21:3<203::AIDSMJ102>3.0.CO;2-K.

Iansiti, M. The New Operational Dynamics of Business Ecosystems: Implications for Policy, Operations and Technology Strategy / M. Iansiti, R. Levien.[S. 1.] : Harvard Business School Working Paper, 2002. $-113 \mathrm{p}$.

Jiang, Hua. The Application of Artificial Neural Networks in Risk Assessment on High-Tech Project Investment / Hua Jiang // International Conference on Business Intelligence and Financial Engineering. - [S. 1. : s. n.], 2009. - P. 17-20.

Kapoor, R. Coordinating and competing in ecosystems: how organizational forms shape new technology investments / R. Kapoor, J. M. Lee // Strategic Management Journal. - 2013. - №34 (3). - P. 274 296. DOI: https://doi.org/10.1002/smj.2010.

Learning and Clustering of Fuzzy Cognitive Maps for Travel Behaviour Analysis / M. Leon [et al.] // Knowledge and Information Systems. - 2014. № 39 (2). - P. 435-462. - DOI: https://doi.org/ 10.1007/s10115-013-0616-z.

Misra, J. Artificial Neural Networks in Hardware: A Survey of Two Decades of Progress / J. Misra, 
I. Saha // Neurocomputing. - 2010. - Vol. 74, № 1-3. - P. 239-255. - DOI: https://doi.org/ 10.1016/j.neucom.2010.03.021.

Pukala, R. Use of Neural Networks in Risk Assessment and Optimization of Insurance Cover in Innovative Enterprises' / R. Pukala // Economics and Management. - 2016. - Vol. 8, № 3. - P. 43 56. - DOI: https://doi.org/10.1515/emj-2016-0023.

Sarcia, S. A. A Statistical Neural Network Framework for Risk Management Process: From the Proposal to its Preliminary Validation for Efficiency/S. A. Sarcia, G. Cantone, V. R. Basili // International Conference on Software and Data Technologies (ICSOFT 2007). Barcelona, Spain. - 2007. - Vol. 6. - P. 46-51.

Sarcia, A. S. Using Artificial Neural Networks to Improve Risk Management Process. TR06, ESEG-DISP/ A. S. Sarcia, G. Cantone. - [S. 1] : University of Roma Tor Vergata, 2006.

Walley, K. Coopetition: an Introduction to the Subject and an Agenda for Research / K. Walley // International Studies of Management \& Organization. - 2007. № 37 (2). - P. 11-31.

Zhang, G. P. Forecasting with Artificial Neural Networks: The State of the Art / G. P. Zhang, E. B. Patuwo, H. Y. Michael // International Journal of Forecasting. - 1998. - Vol. 14, № 1.P. 35-62. - DOI: https://doi.org/10.1016/S01692070(97)00044-7.

\section{REFERENCES}

Kachalov R.M., Sleptsova Yu.A. Dinamika riska v protsessakh innovatsionnogo razvitiya predpriyatiy [Dynamics of Risk in the Processes of Innovative Development of Enterprises]. Vestnik Volgogradskogo gosudarstvennogo universiteta. Seriya 3. Ekonomika. Ekologiya [Science Journal of Volgograd State University. Global Economic System], 2014, no. 4 (27), pp. 72-86. DOI: http://dx.doi.org/10.15688/ jvolsu3. 2014.4.8.

Kachalov R.M., Sleptsova Yu.A. Strukturirovanie sistemno-ekonomicheskogo prostranstva predpriyatiya $\mathrm{v}$ zadachakh upravleniya urovnem riska [Structuration of System and Economic Space of Enterprise and Risk Management]. Rossiyskiy zhurnal menedzhmenta [Russian Management Journal], 2015, vol. 13, no. 4, pp. 69-84.

Kachalov R.M. Upravlenie ekonomicheskim riskom: teoreticheskie osnovy i prilozheniya [Managing Economic Risk: Theoretical Background and Applications]. Saint Petersburg, Nestor-Istoriya Publ., 2012. 288 p.
Kleiner G.B. Sistemnaya ekonomika kak platforma razvitiya sovremennoy ekonomicheskoy teorii [System Economics as a Platform for Development of Modern Economic Theory]. Voprosy Ekonomiki, 2013, no. 6, pp. 428. DOI: https://doi.org/10.32609/0042-87362013-6-4-28.

Polterovich V.M. Instituty dogonyayushchego razvitiya ( $\mathrm{k}$ proektu novoy modeli ekonomicheskogo razvitiya Rossii) [Institutions of Catching-Up Development (On the Project of a New Model for Economic Development of Russia)]. Ekonomicheskie $i$ sotsialnye peremeny: fakty, tendentsii, prognoz [Economic and Social Changes: Facts, Trends, Forecast], 2016, no. 5 (47), pp. 34-56.

Romanovskiy A.V., Shokin Ya.V. Neyroekonomika i ee integratsiya $\mathrm{v}$ ekonomicheskuyu nauku [Neuroeconomy and Its' Integration to the Economical Science]. Ekonomicheskie nauki [Economic Sciences], 2010, no. 4, pp. 42-45.

Oehmen J., Olechowski A., Robert Kenley C., BenDaya M. Analysis of the Effect of Risk Management Practices on the Performance of New Product Development Programs. Technovation, Elsevier Ltd., 2014, vol. 34, no. 8, pp. 441-453. DOI: https://doi.org/10.1016/ j.technovation.2013.12.005.

Boland J.R.J., Lyytinen K., Yoo Y. Wakes of Innovation in Project Networks: The Case of Digital 3-D Representations in Architecture, Engineering, and Construction. Organization Science, 2007, no. 18 (4), pp. 631-647. DOI: https://doi.org/ 10.1287/orsc.1070.0304.

COSO: Enterprise Risk Management - Integrating with Strategy and Performance, 2017. URL: https://www.coso.org/Pages/erm.aspx (accessed 5 June 2019).

Das T., Teng B. Risk Types and Inter-Firm Alliance Structures. Journal of Management Studies, 1996, no. 33 (6), pp. 827-843. DOI: https://doi.org/ 10.1111/j.1467-6486.1996.tb00174.x.

Das T., Teng B. Trust, Control, and Risk in Strategic Alliances: An Integrated Framework. Organization Studies, 2001, no. 22 (2), pp. 251-283.

Azadeh A., Saberi M., Noorossana R., Mehrabad M.S., Anvari M., Izadbakhsh H. Estimating Efficient Value of Controllable Variable Using an Adaptive Neural Network Algorithm: Case of a Railway System. Journal of Scientific and Industrial Research, 2012, no. 71 (1), pp. 45-50.

Gawer A., Cusumano M.A. Industry Platforms and Ecosystem Innovation. Journal of Product Innovation Management, 2014, no. 31 (3), pp. 417-433. DOI: https://doi.org/10.1111/ jpim. 12105 . 
Gulati R., Nohria N., Zaheer A. Strategic Networks. Strategic Management Journal, 2000, no. 21 (3), pp. 203-215. DOI: https://doi.org/10.1002/ (SICI) 1097-0266(200003)21:3<203::AIDSMJ102>3.0.CO;2-K.

Iansiti M., Levien R. The New Operational Dynamics of Business Ecosystems: Implications for Policy, Operations and Technology Strategy. Harvard Business School Working Paper, September 2002. 113 p.

Jiang Hua. The Application of Artificial Neural Networks in Risk Assessment on High-Tech Project Investment. International Conference on Business Intelligence and Financial Engineering, 2009, pp. 17-20.

Kapoor R., Lee J.M., Coordinating and Competing in Ecosystems: How Organizational Forms Shape New Technology Investments. Strategic Management Journal, 2013, no. 34 (3), pp. 274296. DOI: https://doi.org/10.1002/smj.2010.

Leon M., Mkrtchyan L., DepaireB., Ruan D., VanhoofK. Learning and Clustering of Fuzzy Cognitive Maps for Travel Behaviour Analysis. Knowledge and Information Systems, 2014, no. 39 (2), pp. 435-462. DOI: https://doi.org/ 10.1007/s10115-013-0616-z.

Misra J., Saha I. Artificial Neural Networks in Hardware: A Survey of Two Decades of Progress.
Neurocomputing, 2010, vol. 74, no. 1-3, pp. 239255. DOI: https://doi.org/10.1016/j.neucom. 2010.03.021.

Pukala R. Use of Neural Networks in Risk Assessment and Optimization of Insurance Cover in Innovative Enterprises. Economics and Management, 2016, vol. 8, no. 3, pp. 43-56. DOI: 10.1515/emj-2016-0023.

Sarcia S.A., Cantone G., Basili V.R. A Statistical Neural Network Framework for Risk Management Process: From the Proposal to Its Preliminary Validation for Efficiency. International Conference on Software and Data Technologies (ICSOFT 2007). Barcelona, Spain, 2007, vol. 6, pp. 46-51.

Sarcia A.S., Cantone G. Using Artificial Neural Networks to Improve Risk Management Process. TR06, ESEG-DISP. University of Roma Tor Vergata, 2006.

Walley K. Coopetition: An Introduction to the Subject and an Agenda for Research. International Studies of Management \& Organization, 2007, no. 37 (2), pp. 11-31. DOI: https://www.jstor.org/ stable/40397695.

Zhang G.P., Patuwo E.B., Michael Y.H. Forecasting with Artificial Neural Networks: The State of the Art. International Journal of Forecasting, 1998, vol. 14, no. 1, pp. 35-62. DOI: https://doi.org/ 10.1016/S0169-2070(97)00044-7.

\section{Information About the Authors}

Roman M. Kachalov, Doctor of Sciences (Economics), Professor, Head of the Laboratory for Publishing and Marketing Activities, Central Economic Mathematical Institute of the Russian Academy of Sciences, Prosp. Nakhimovskiy, 47, 117418 Moscow, Russian Federation; Professor, Department of Digital Economics, Dubna State University, Universitetskaya St., 19, 141982 Dubna, Russian Federation, kachalov1ya@ya.ru, https://orcid.org/0000-0001-5866-3390

Yulia A. Sleptsova, Candidate of Sciences (Economics), Associate Professor, Senior Researcher, Laboratory for Publishing and Marketing Activities, Central Economic Mathematical Institute of the Russian Academy of Sciences, Prosp. Nakhimovskiy, 47, 117418 Moscow, Russian Federation; Associate Professor, Department of Digital Economics, Dubna State University, Universitetskaya St., 19, 141982 Dubna, Russian Federation, julia_sleptsova@mail.ru, https://orcid.org/0000-0001-9343-3574

Yan V. Shokin, Doctor of Sciences (Economics), Associate Professor, Department of Digital Economics, Dubna State University, Universitetskaya St., 19, 141982 Dubna, Russian Federation, yshokin@mail.ru, https://orcid.org/0000-0001-9772-650X

\section{Информация об авторах}

Роман Михайлович Качалов, доктор экономических наук, профессор, заведующий лабораторией издательской и маркетинговой деятельности, Центральный экономико-математический институт РАН, просп. Нахимовский, 47, 117418 г. Москва, Российская Федерация; профессор кафедры цифровой экономики, Государственный университет «Дубна», ул. Университетская, 19, 141982 г. Дубна, Российская Федерация, kachalov1 уа@ya.ru, https://orcid.org/0000-0001-5866-3390 
Р.М. Качалов, Ю.А. Слепц̧ова, Я.В. Шокин. Оценка риска реализации инновационных проектов предприятий

Юлия Анатольевна Слепцова, кандидат экономических наук, доцент, старший научный сотрудник лаборатории издательской и маркетинговой деятельности, Центральный экономико-математический институт РАН, просп. Нахимовский, 47, 117418 г. Москва, Российская Федерация; доцент кафедры цифровой экономики, Государственный университет «Дубна», ул. Университетская, 19, 141982 г. Дубна, Российская Федерация, julia_sleptsova@mail.ru, https://orcid.org/0000-0001-9343-3574

Ян Вячеславович Шокин, доктор экономических наук, доцент кафедры цифровой экономики, Государственный университет «Дубна», ул. Университетская, 19, 141982 г. Дубна, Российская Федерация, yshokin@mail.ru, https://orcid.org/0000-0001-9772-650X 